\title{
Perichondrial Cutaneous Grafts in Facial Reconstruction
}

\author{
1Jamie G Bizzell, 2Jennings R Boyette
}

\begin{abstract}
Aim: The purpose of this study is to review the indications, techniques, advantages, and outcomes of perichondrial cutaneous grafts (PCCG).
\end{abstract}

Background: The PCCG is a composite graft utilized in the reconstruction of select head and neck defects. This graft, much studied and popularized by Dr Frederick Stucker, has several intrinsic benefits due to its size, thickness, contour, and unique perichondrial layer.

Results: The PCCG is primarily utilized in the reconstruction of nasal and lower eyelid defects. This graft has been demonstrated to yield superior results to a full-thickness skin graft (FTSG) in these cosmetically sensitive locations and has the convenience of a single-stage procedure that may be performed under local anesthesia in contrast to a paramedian forehead flap (PMFF). Animal studies have shown less contraction in comparison to FTSGs. Large case series have reported low rates of graft loss and good esthetic outcomes.

Conclusion: The PCCG is an option to consider in the reconstruction of select head and neck defects, as it tends to offer excellent cosmetic results and minimal morbidity.

Clinical significance: The PCCG has several advantages over a FTSG for moderate to large defects of the face, and is an excellent reconstructive option for nasal defects in which a PMFF may not be a viable option.

Keywords: Composite graft, Head and neck reconstruction, Nasal reconstruction, Perichondrial cutaneous grafts, Skin graft.

How to cite this article: Bizzell JG, Boyette JR. Perichondrial Cutaneous Grafts in Facial Reconstruction. Int J Head Neck Surg 2016;7(3):182-187.

Source of support: Nil

Conflict of interest: None

\section{INTRODUCTION}

The perichondrial cutaneous graft (PCCG) is a composite graft that has a variety of advantages that make it an ideal candidate for reconstruction of certain facial skin defects. Brent and $\mathrm{Ott}^{1}$ first described the utilization of the PCCG

\footnotetext{
${ }^{1}$ Resident, ${ }^{2}$ Assistant Professor

${ }^{1,2}$ Department of Otolaryngology, University of Arkansas for Medical Sciences, Little Rock, Arkansas, USA

Corresponding Author: Jennings R Boyette, Assistant Professor, Department of Otolaryngology, University of Arkansas for Medical Sciences, Little Rock, Arkansas, USA, Phone: +5016865140, e-mail: jrboyette@uams.edu
}

for reconstruction of facial defects in 1978. Since this time, the value of this graft has been confirmed and expanded. The popularization of this graft in head and neck reconstruction can much be contributed to Dr Frederick Stucker, as he has reported extensively on the characteristics of this graft in a large number of patients.

There are many options for reconstruction in the face depending on the size and location of the defect. Flap or graft reconstruction is often required after traumatic events to the face and following resection of cutaneous malignancies. Although there are many alternatives for reconstruction in the head and neck, all options come with their inherent benefits and disadvantages. For example, the paramedian forehead flap (PMFF) is an excellent reconstructive option for large defects of the nose. However, the PMFF involves a two-stage procedure with an overtly visible pedicle in the interim. In addition, many patients may have future malignant lesions that will ultimately require resection and reconstruction, and thus, the PMFF may need to be reserved for future surgeries. Therefore, other alternatives, such as the PCCG, necessitate consideration when planning the reconstruction of facial defects.

The PCCG comprises epidermis, dermis, a layer of subcutaneous tissue, and perichondrium. The portion of this graft that makes it distinct from others lies within the perichondrium. Perichondrium has been demonstrated both to have chondrogenic potential and to aid in healing through fibroblastic activity. Specifically, Duynstee et $\mathrm{al}^{2}$ demonstrated that the inner layer (cambium) is responsible for chondrogenic potential, while the outer layer helps the wound edge heal through proliferation of fibroblasts. Perichondrium also contains a vascular plexus, which may contribute to the rapid revascularization process seen with a PCCG. ${ }^{3}$ Furthermore, the perichondrial layer provides a barrier between the deep dermis and the recipient bed, which may help minimize graft contraction, thickening, and fibrosis. Portuese et $\mathrm{al}^{3}$ speculated that this barrier may decrease fibroblast proliferation after comparing the PCCG with the traditionally used full-thickness skin graft (FTSG). Portuese and other authors have also reported that these grafts can exhibit neocartilage formation, although this has not been definitively seen in humans. ${ }^{3,4}$ Possibly associated with this concept, Stucker and Shaw ${ }^{5}$ report growth of these grafts with children as they age. Given the unique qualities of this graft, the objective of this review is to 
outline the indications, technique, outcomes, and distinct advantages of the PCCG.

\section{RESULTS}

The inherent advantages of the graft and indications for its use have been reported by several authors. A study conducted by Portuese et $\mathrm{al}^{3}$ demonstrated the distinct advantages of the PCCG in contrast to the traditionally employed FTSG. In rabbit models, the PCCG retained its circular shape and increased its surface area by $44 \%$, whereas the FTSG contracted in size by $19 \%$ and changed to an ovoid orientation. In addition, PCCG had a higher success rate at graft take vs FTSG ( $85 \%$ vs $65 \%$ ), preserved its epidermal appendages, and maintained its thickness. In addition, all models demonstrated neocartilage formation. ${ }^{3}$

The PCCG can be utilized for the reconstruction of head and neck defects, particularly for the lower $1 / 3$ of the nose, lower eyelid, and auricle (Figs $1 \mathrm{~A}$ and $\mathrm{B}$ and 2A to C). In a series published by Stucker in 1992, 96/112 cases involved nasal reconstruction, 10/112 cases utilized PCCG for eyelid repair, and 6/112 cases were completed for auricular defects. ${ }^{5}$ However, using the PCCG for auricular defects has fallen out of favor, as a FTSG in this location yields similar results. ${ }^{5}$

\section{Graft Harvest}

Although techniques vary, the most traditional and widely used technique involves harvesting the graft consisting of skin, dermis, subcutaneous tissue, and a layer of perichondrium from the anterior conchal bowl. The surgeon should first make a template of the defect and mark the conchal bowl based on this template;
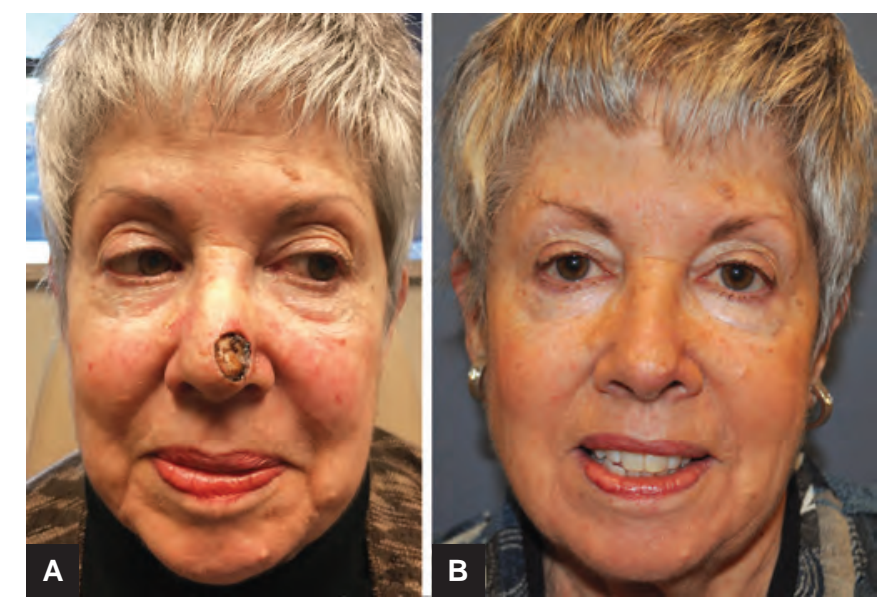

Figs $1 \mathrm{~A}$ and $\mathrm{B}$ : Patient with a left nasal tip defect: (A) Patient elected to undergo PCCG instead of flap reconstruction; and (B) postoperative result at 3 months after reconstruction with a PCCG.

$1 \%$ lidocaine with 1:100,000 epinephrine is then injected into the anterior conchal bowl just above the conchal cartilage in order to elevate the perichondrium through hydrodissection..$^{4-7}$ Stucker and Shaw ${ }^{5}$ advocate for performing this injection in the central portion of the conchal bowl, where they speculate the perichondrium is more adherent to the skin in order to prevent separation of the perichondrium and skin with the injection. Gloster et al ${ }^{4}$ do not consider the location of the injection as critical. But others recommend that if the injection is performed in the incorrect plane superficial to the perichondrium, then the surgeon should utilize the contralateral ear to obtain the graft..$^{5-7}$ Alternatively, local can be infiltrated circumferentially around the planned graft donor site in order to avoid injection into the incorrect plane. After injection, the incision is started 2 to $3 \mathrm{~mm}$ inferior to the
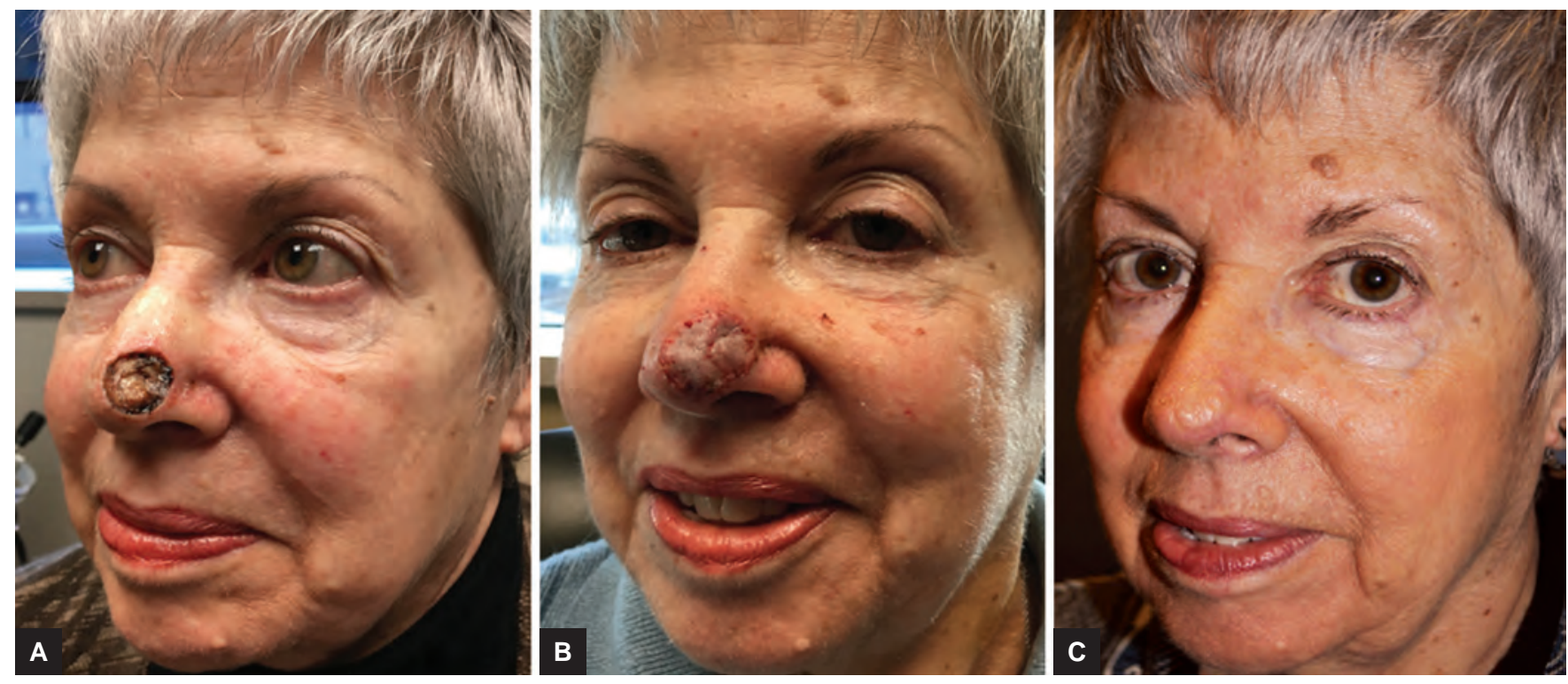

Figs 2A to C: (A) Left nasal tip defect prior to reconstruction; (B) appearance of PCCG after 1 week (note the slightly grayish appearance which is typical of this graft); and (C) postoperative outcome at 3 months 
apex of the antihelix, antitragus, and inferior crus and continued within the conchal bowl to accommodate the size of the defect. The incision is made to the depth of the cartilage, and a subperichondrial dissection is then performed using a periosteal elevator. The dissection is then taken forward to near the external auditory canal. $^{4-7}$

Once the graft is harvested, it is trimmed meticulously to match the size of the defect and placed in the recipient bed (Figs 3A to D). At this point, most prefer to inset the graft with interrupted nonabsorbable sutures ${ }^{4-7}$ Stucker promotes using interrupted 5-0 chromic sutures to secure the graft into the recipient bed and to allow drainage sites. ${ }^{5-7}$ However, Gloster places a bolster over his graft with similar results. ${ }^{4}$

The reported outcomes for this technique are excellent. Gloster et al repaired 15 nasal tip or ala defects with an anteriorly harvested PCCG and reported excellent cosmesis without incidences of graft necrosis, failure, or distortion. Scar revision or dermabrasion were not required in any of these cases. ${ }^{4}$ Stucker has published several series reporting his outcomes. In his paper in 1992, the success rate of PCCG in this series was $98 \%$ (110/112 cases). The majority of his patients (101/112) were noted to have "excellent cosmetic results," while 9/11 of the poor cosmetic results could be attributed to surgical technique. ${ }^{5}$ Stucker published another series in 2008 on PCCG results in 406 patients with ages ranging between 7 days and 94 years old. One hundred and seventy of these grafts were harvested to reconstruct defects secondary to trauma, while 236 were used to reconstruct defects following skin cancer resection. In this cohort, there were four $(0.99 \%)$ total graft failures, all occurring in patients with smoking history. There were also six partial graft losses, defined as loss of graft by less than $30 \%$. The remaining grafts were noted to have no evidence of contraction, and the grafts placed in children continued to grow as the child developed. ${ }^{6}$ In addition, Patterson et al performed a case review in which a PCCG was utilized for reconstruction of facial defects, and only $1 / 41$ graft failures were reported and 39/41 of the cases had good or excellent cosmetic results. One poor cosmetic result was associated with the graft failure, while the other was from notching at the alar margin. ${ }^{8}$

In contrast to the anterior approach for graft harvest, the posterior approach of graft harvest has also been described. Kalbermatten et $\mathrm{al}^{9}$ utilized this approach in order to simplify closure of the donor site. Other variations in their technique include harvesting only the outer layer of perichondrium, and employing graft cooling for the first 4 days postoperatively. The cooling was completed in order to decrease metabolic tissue demands, as there was a concern for graft survival since the posterior auricular skin has a slightly thicker subcutaneous layer and a looser connection between skin and perichondrium. Despite the differences, the results were comparable to previous studies. Three of 14 patients in their series had partial necrosis at 2 weeks with one having signs of infection prior to the tissue necrosis. Despite these initial results, all 14 demonstrated excellent final cosmesis without contraction or depression at 6 months with only $1 / 14$ grafts displaying hyperpigmentation. Furthermore, a modified tension spring balance analyzed the compression force of each ala, and the repaired nasal ala was essentially comparable in strength to the native ala. ${ }^{9}$ Schmid et al also varied the traditional technique on one patient by employing the posterior approach and harvesting a strip of cartilage with the PCCG to reconstruct the alar rim in their patient with a defect involving $>50 \%$ of the alar rim. This single patient had $100 \%$ graft take and excellent cosmetic results. ${ }^{10}$

\section{DONOR SITE CLOSURE}

Historically, the donor site was closed by resecting the exposed cartilage followed by skin graft closure, as described by Brent and Ott. ${ }^{1}$ However, with the increasing utilization of local flaps, the postauricular interpolated island flap, or "flip-flop" flap, gained popularity in
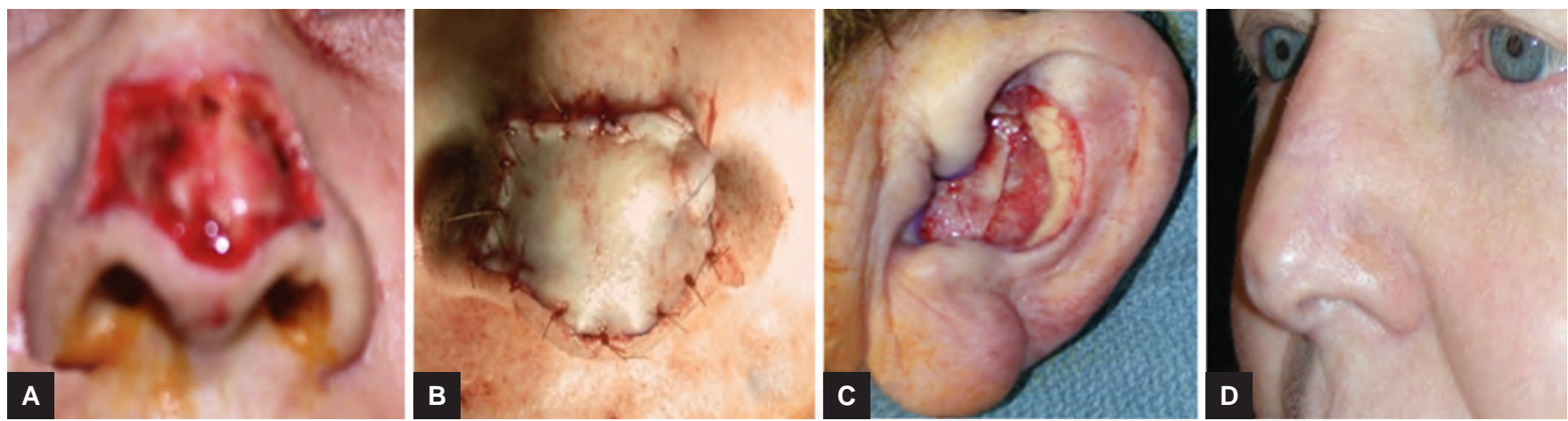

Figs 3A to D: Nasal defect amenable to PCCG reconstruction: (A) Defect extended to encompass tip esthetic subunit; (B) PCCG sutured in place with interrupted sutures; (C) donor site defect at conchal bowl; and (D) early postoperative appearance of healed graft. Courtesy: Dr Frederick Stucker 
closing this defect (Figs 4A to F). In order to perform this closure, a window of conchal cartilage is first resected. Stucker reports the resection of approximately $1 \mathrm{~cm}$ of cartilage to be sufficient. The pinna is then retracted anteriorly, and the postauricular muscle is identified. The center of the flap is then localized to the intersection of the postauricular sulcus and the postauricular muscle's insertion. An incision is made through the skin and subcutaneous tissues, leaving $50 \%$ of the base attached. The flap is then rotated $180^{\circ}$ through the window of resected cartilage. The flap is then sutured into place anteriorly with interrupted sutures and several mattress sutures can be placed to close any dead space. Posteriorly, the skin is undermined and closed primarily in a vertical orientation. ${ }^{4-7}$

Stucker reports excellent results from closure with the postauricular flip-flop flap (PAFFF). In his series, only 4/354 cases had suboptimal results when the conchal defect was closed utilizing the PAFFF. In two cases, partial necrosis of the PAFFF was documented, and dehiscence of the postauricular closure was noted in the other two cases. Despite these initial failures, with routine wound care, the conchal bowl healed equivalently to the other cases in the series. Fifteen patients reported pain for greater than 1 week, which the author contributed to perichondritis. ${ }^{7}$ Patterson et al also report good outcomes utilizing the flip-flop flap with no cases of flap failure or infection. However, they do report two cases of postauricular wound dehiscence, which healed without further intervention. ${ }^{8}$

Another method to close the donor site includes allowing it to heal by secondary intention. Utilizing this approach, the defect will typically reepithelialize in 3 to 5 weeks. Gloster and Broadland ${ }^{4}$ recommends removing cartilage using a $2 \mathrm{~mm}$ punch in order to allow granulation from the posterior aspect of the cartilage to facilitate healing.

The posterior approach for PCCG harvest was initially developed in order to decrease the complexity and complications associated with donor site closure. When the posterior approach is utilized, small defects can be closed primarily or a local transposition flap can be used to close the defect. This closure is discreetly located in the postauricular area and resulted in excellent cosmesis without reports of dehiscence, infection, or other
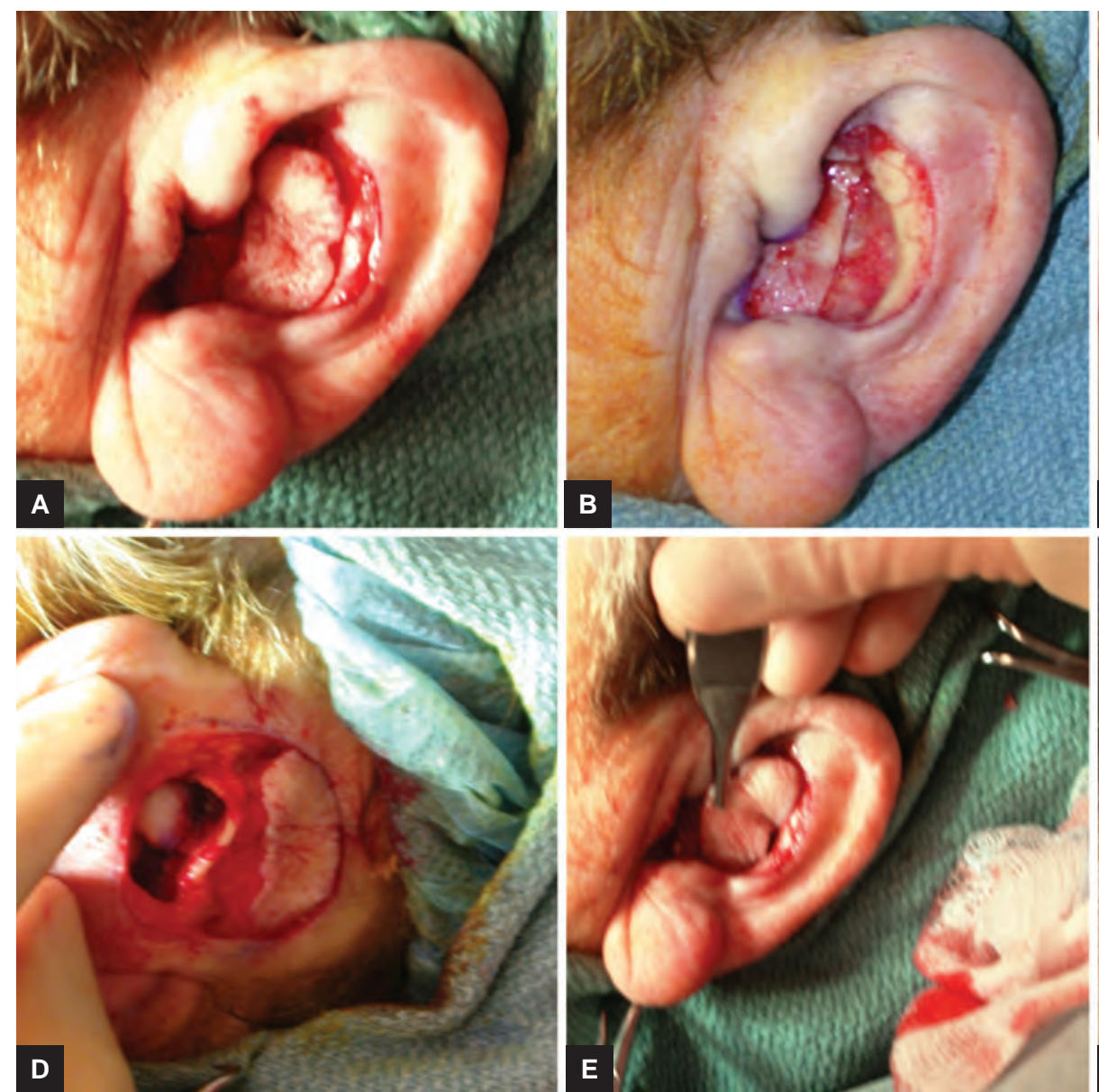
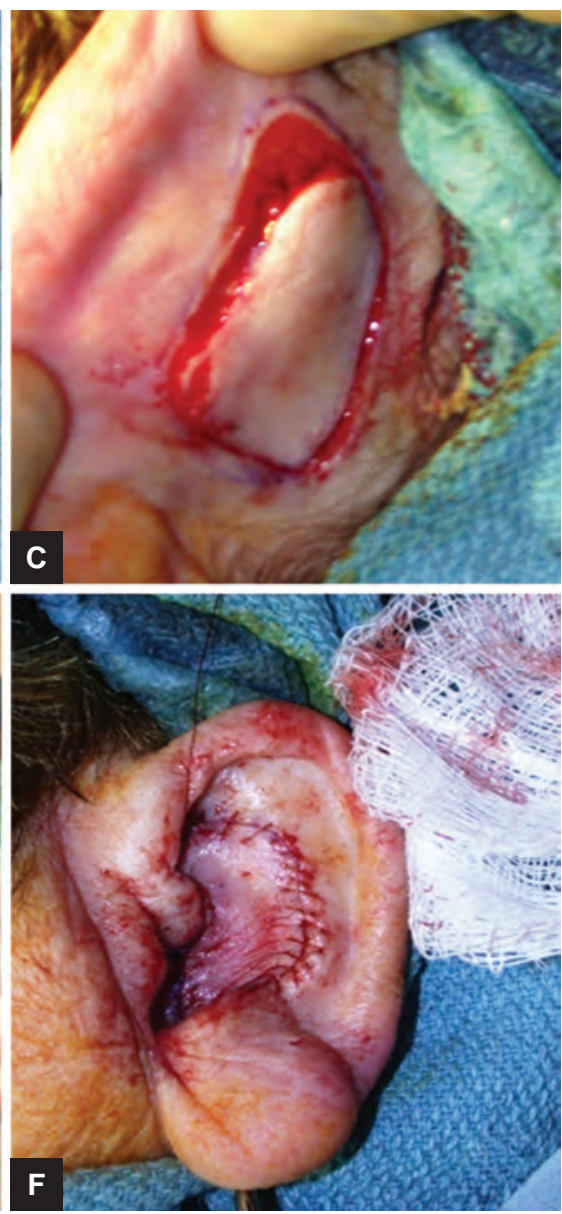

Figs 4A to F: Donor site reconstruction of patient in Figure 3: (A) PCCG to be excised from conchal bowl; (B) defect following graft removal; (C) postauricular island flap based upon postauricular muscle; (D) window of cartilage removed to accommodate PAFFF; (E) flap rotated through defect into position; and (F) flap sutured into position. The secondary defect from the flap is reconstructed by advancement of remaining postauricular skin. Courtesy: Dr Frederick Stucker 
complications. In addition, patients did not report prolonged postoperative pain. ${ }^{9}$

\section{DISCUSSION}

As a result of the unique features of the PCCG, several indications for its use exist. Specifically, the PCCG has been utilized for auricular defects, periorbital defects, and nasal defects. The PCCG is ideal for reconstructing the lower $1 / 3$ of the nose, as an entire subunit can be reconstructed with this graft if needed. ${ }^{4,6}$ Other indications include defects in children less than $4 \mathrm{~cm}^{2}$, ectropion, anterior lamella trauma, lower eyelid defects, and for use in patients who prefer single-stage procedures. There are minimal reported contraindications to PCCG including local conchal pathology, markedly depressed defects, or following a technical error of injecting local anesthesia superficial to the perichondrium. In the latter situation, the case can be completed by harvesting the contralateral PCCG. ${ }^{6}$

The PCCG has many qualities that make it distinct from other reconstructive options for facial defects. The PCCG in rabbit models all demonstrated neocartilage formation, and this quality may provide additional strength to the graft and contribute to the absence of contraction seen in these grafts. ${ }^{3}$ In addition to the potential for neocartilage formation, children are ideal candidates for PCCG, as skin grafts will often yield unsatisfactory results in facial defects, and children's skin does not contain the laxity often required for local flaps. ${ }^{6}$ The epidermal portion of the PCCG also more accurately matches the color and sebaceous quality of the nose when compared to some FTSGs. ${ }^{4}$ The PCCG is also an intrinsically thicker graft, which contributes to its ability to better accommodate some deeper defects. Also as mentioned previously, the PCCG contracts to a lesser degree and maintains its thickness in contrast to the FTSG. In addition to the inherent advantages of the graft itself, the PCCG is a relatively simple procedure, and it may be performed as an outpatient procedure in a single stage under local anesthesia. ${ }^{5}$ This benefit is in direct contrast to a PMFF, which could also be utilized for reconstruction of similar nasal defects. This advantage not only lends itself to patient preference but also contributes to its ability to potentially save both time and money to the health care system.

As with any procedure, risks are associated with the surgery. Specifically, risks include necrosis of the graft, possibility of graft contraction, graft atrophy, textural changes, infection, and wound healing issues with the donor site. Size constraints are also a concern, as the size of the conchal bowl limits the graft size and thickness. Stucker and Shaw ${ }^{5}$ report that the PCCG can be harvested to a size of $2.5 \mathrm{~cm}$ by $4 \mathrm{~cm}$ without difficulty. Thickness of the PCCG is typically limited to 2 to $3 \mathrm{~mm}$. ${ }^{4}$ However, despite these associated risks and limitations, the PCCG has been reported to have overall excellent outcomes and minimal morbidity.

\section{CONCLUSION}

The PCCG offers an excellent option in the armamentarium of reconstructive options for facial defects, particularly the lower $1 / 3$ of the nose and the lower eyelid. Many patients with larger skin defects do not wish to undergo a PMFF, yet desire a cosmetically acceptable outcome. The PCCG is an excellent option for these patients, as well as patients with the preponderance for developing skin cancers in which a PMFF may be reserved for a future cancer defect (or is unable to be utilized due to cutaneous disease on the forehead itself). Overall, these grafts have excellent cosmetic results with minimal morbidity. In addition, the perichondrial layer offers a distinct advantage in the utilization of this graft with its potential for neocartilage formation and ability to grow in children as they mature. Furthermore, the PCCG is a fairly straightforward procedure that can be performed as a single-stage outpatient surgery and has demonstrated superior cosmetic results to a FTSG.

\section{CLINICAL SIGNIFICANCE}

The PCCG has been shown to be an excellent reconstructive option in select head and neck defects, including the lower $1 / 3$ of the nose and the lower eyelid. This graft should be considered in reconstructing facial defects, as it yields excellent cosmetic results, and can be performed under local anesthesia in a single-stage procedure, with minimal morbidity.

\section{ACKNOWLEDGMENTS}

The authors would like to acknowledge the significant contribution Dr Frederick Stucker has made to the field of facial plastic and reconstructive surgery. This review highlights the impact he has made on our field by examining just one of the many surgical techniques his work has influenced.

\section{REFERENCES}

1. Brent B, Ott R. Perichondro-cutaneous graft. Plast Reconstr Surg 1978 Jul;62(1):1-14.

2. Duynstee ML, Verwoerd-Verhoef HL, Verwoerd CD, Van Osch GJ. The dual role of perichondrium in cartilage wound healing. Plast Reconstr Surg 2002 Sep;110(4):1073-1079.

3. Portuese W, Stucker F, Grafton W, Shockley W, Gage-White L. Perichondrial cutaneous graft: an alternative in composite skin grafting. Arch Otolaryngol Head and Neck Surg 1989 Jun;115(6):705-709. 
4. Gloster HM, Brodland DG. The use of perichondrial cutaneous grafts to repair defects of the lower third of the nose. Br J Dermatol 1997 Jan;136(1):43-46.

5. Stucker FJ, Shaw GY. The perichondrial cutaneous graft: a 12-year clinical experience. Arch Otolaryngol Head Neck Surg 1992 Mar;118(3):287-292.

6. Stucker FJ, Walsh WE, Dammert M, Lian T. The perichondrial cutaneous graft: a facial reconstructive option for the ages (ages 1 week to 94 years). Laryngoscope 2008 Oct;118(10):1753-1757.

7. Stucker FJ, Sanders KW. A method to repair auricular defects after perichondrial cutaneous grafting. Laryngoscope 2002 Aug;112(8 Pt 1):1384-1386.
8. Patterson AR, Brady G, Walker PD, Telfer MR. The perichondrial cutaneous graft and "flip-flop" flap in facial reconstruction: a series of 41 cases. Br J Oral Maxillofacial Surg 2008 Mar;46:114-118.

9. Kalbermatten DF, Haug M, Wettstein R, Schaefer DJ, Pierer G. New posterior auricular perichondrial cutaneous graft for stable reconstruction nasal defects. Aesth Plast Surg 2005 Nov-Dec;29(6):489-495.

10. Schmid DW, Summa PG, Wettstein R, Erba P, Wassim R, Kalbermatten DF. Posterior auricular perichondrial cutaneous graft combined with cartilage strip in nostril reconstruction. Eplasty 2008 Aug;8:e42. 